CORRECTION

https://doi.org/10.1038/s41586-018-0489-0

\title{
Publisher Correction: Challenging local realism with human choices
}

The BIG Bell Test Collaboration

Correction to: Nature https://doi.org/10.1038/s41586-018-0085-3, published online 09 May 2018.

In the HTML version of this Letter, the corresponding author link (M. W. Mitchell; morgan.mitchell@icfo.eu) was missing and the citation file was empty. The list of consortium authors was duplicated, with spurious superscript numbers, in the Acknowledgements (the PDF version was correct). In addition, in Fig. 2a of this Letter, the label '4', which indicates Vienna in the inset, was inadvertently displaced into Siberia in the main panel. These errors have been corrected online. 